\title{
Pathway to mental health recovery: a qualitative and quantitative study on the needs of Chinese psychiatric inpatients
}

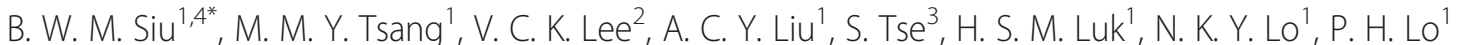
and Y. L. Leung ${ }^{1}$

\begin{abstract}
Background: Exploration of the information and participation needs of psychiatric inpatients is an important step for the implementation of recovery-oriented mental health service. The objective of this study was to explore the information and participation needs of Chinese psychiatric inpatients in the largest psychiatric hospital in Hong Kong.

Methods: The study was divided into two parts. In the first part, eight focus groups with patients, patients' relatives and healthcare professionals were held to identify 22 items of information needs and 16 items of participation needs of Chinese psychiatric inpatients. Basing on the items identified in the first part of the study, a questionnaire was developed to survey on the importance of the different information and participation needs in the second part of the study. Participants were asked to rate in rank order their perceived importance of the items in the questionnaire survey.

Results: A hundred and eighty three Chinese psychiatric inpatients completed the questionnaire and the majority of them suffered from schizophrenia $(68.3 \%)$. For information needs, the top three needs rated by patients as the most important in descending order were: "Information on the classifications of mental illnesses, signs and symptoms and factors contributing to relapse", "Information on the criteria and arrangements for discharge", and "Information on the importance of psychiatric drug taking and its side effects". For participation needs, the top three needs rated by patients as the most important in descending order were: "Enquiring about personal needs and arrangements", "Keeping in touch with the outside world", and "Learning and practising self-management".

Conclusions: This study reveals that Chinese psychiatric inpatients are concerned about information on their mental illness and its treatments as well as the criteria for discharge. On the other hand, patients are concerned about their personal needs, their self-management, as well as their keeping in touch with the outside world during their hospitalisation. Moreover, patients with different socio-demographic and clinical characteristics have different information and participation needs. The results of the present study serve as a reference for designing guidelines, strategies, and programmes to meet the information needs and participation needs of psychiatric inpatients in Hong Kong.
\end{abstract}

Keywords: Information, Participation, Mental Health Recovery, Inpatients, Chinese

\footnotetext{
* Correspondence: bonniew114m@yahoo.com

'Department of Psychiatry, Castle Peak Hospital, Hong Kong, SAR, People's

Republic of China

${ }^{4}$ Department of Forensic Psychiatry, Castle Peak Hospital, 15 Tsing Chung

Koon Road, Tuen Mun, New Territories, Hong Kong

Full list of author information is available at the end of the article
} 


\section{Background}

\section{Mental health recovery}

Mental health recovery is a journey of healing and transformation enabling a person with a mental disability to live a meaningful life in the community of his or her choice, while striving to achieve his or her full potential [1]. As an essential direction of mental health care reform and a move towards continuous service quality improvement, the concept of recovery-oriented care has already been incorporated into the national policies, service standards and guiding principles for the transformation of the mental health systems in many countries, including the United Kingdom, New Zealand, Australia, America, and Canada [2-7].

\section{Recovery-oriented services}

Recent systematic review strongly supports the view that hospital-based facilities for individuals with severe mental illnesses should deliver "recovery-oriented" services [8]. Moreover, there is evidence supporting the idea that implementation of recovery-oriented services could increase the satisfaction of patients towards the services delivered to them $[9,10]$. William Anthony has proposed a widely quoted definition of recovery as 'a deeply personal, unique process of changing one's attitudes, values, feelings, goals, skills and roles. Recovery involves the development of a new meaning and purpose in one's life as one grows beyond the catastrophic effects of mental illness' ([11], p.21). This definition of recovery emphasised that recovery is an individualised process and that patients should be empowered, and their strengths should be explored in order for them to gain a new valued role in life despite the detrimental effects of mental illness. Also, as recovery is a personal and individualised process, patient's participation in the treatment and management plan is important for the development of a treatment plan tailor-made for the patient. Farkas and colleagues concurred with the definition of recovery by William Anthony that recovery-oriented evidence-based mental health programmes should be grounded on the notion that a majority of people can grow beyond the catastrophe of a severe mental illness and lead a meaningful life in their own community [12]. Among the different strategies in the implementation of recovery-oriented services, the promotion of "patient empowerment" and "patient's participation" are perceived to be of upmost importance [1, 13-15].

\section{Information and participation needs}

"Patient empowerment" means "the provision of information regarding therapeutic options so that a patient can actively participate in the decision on whether to undergo a diagnostic or therapeutic procedure, or pursue other alternatives" $[16,17]$. In a focus group study of patients in acute psychiatric wards conducted in the United Kingdom, it was concluded that information is the key to patient empowerment and fulfilling the information needs of psychiatric inpatient is essential for the development of patient-centred care [18]. On the other hand, high quality patient-centred care should address the information needs of psychiatric inpatients. A survey conducted in Sweden on two different samples of psychiatric inpatients revealed that effort should be put on the area of patient information as one of the targets for the development of quality assurance programmes [19]. For "patient's participation", the involvement of patients in organisational service planning and clinical governance processes has been highlighted [8]. In addition to collaboration in their care, patients' participation in developing, running and reviewing services has been shown to have positive outcomes for both the services and the patients who take on these roles $[9,10]$. Addressing the participation needs of psychiatric inpatient has been a major focus for the delivery of quality mental health services in the recent years [20].

\section{Traditional view in Chinese setting}

The traditional healthcare service delivery in the Chinese populations emphasises "paternalism" or "maternalism" and healthcare professionals tend to provide information to psychiatric inpatients basing on their own perspective and prescribe the management plans for patients with the patients being relatively passive and less involved in decisions about his or her own treatment and rehabilitation process [21, 22].

\section{Recovery-oriented services in Asians}

Despite a long history of recovery-oriented services being implemented in mental health settings in a number of countries, recovery-oriented practice is relatively novel in the mental health systems serving Chinese populations. In Asian countries such as mainland China and Taiwan, recent service reform towards community mental health care and the focus on the strengths and resources of patients as well as patients' self-help are in line with the delivery of recovery-oriented mental health service [22-26]. In Hong Kong, an Asian city, it is only in recent years that recovery-oriented practice has been promoted as one of the directions of mental health service reform. To demonstrate this progress, peer specialists, for example, are employed in different mental health units in Hong Kong $[21,27-29]$. Because of the traditional view on healthcare service delivery as mentioned above, despite this progress of employing peer specialists, meeting the information needs and participation needs of psychiatric inpatient is still a challenge for the Chinese populations. The investigation of the information and participation needs of Chinese psychiatric inpatients shall be one of the initial 
steps in fulfilling their needs as well as for the implementation of recovery-oriented mental health services with Chinese populations.

\section{Objective of this study}

As the concept of recovery is relatively new in the Chinese populations and different researchers have different definitions on recovery, it is worthwhile that this concept in the context of information and participation needs be explored in both qualitative and quantitative ways $[1-7,11,12]$.

This study set out to explore the information and participation needs of Chinese psychiatric inpatients by focus group discussion and questionnaire survey in Castle Peak Hospital, the largest psychiatric hospital in Hong Kong. Prior to this study, there had been no local study focusing on the exploration of these areas for psychiatric inpatient.

\section{Methods}

This is a qualitative and quantitative study on the needs of Chinese psychiatric inpatients with focus on their information and participation needs. This study was divided into two parts. The first part (the qualitative part) was to identify a pool of items of information and participation needs of Chinese psychiatric inpatients by focus group discussion involving psychiatric inpatients, patients' relatives and healthcare professionals. A survey questionnaire was then developed basing on the pool of items identified in the focus group discussions. In the second part (the quantitative part) of the study, a group of Chinese psychiatric inpatients were recruited as participants. They were asked to rate in rank order their perceived importance of the items in the survey questionnaire.

\section{Ethics, consent and permissions}

In Hong Kong, Castle Peak Hospital provides inpatient, out-patient, and community psychiatric services for people residing in Tuen Mun area and Yuen Long area serving a population of more than a million. Tuen Mun and Yuen Long constituted the New Territories West Cluster, according to the division of the Hospital Authority of Hong Kong. Approval to conduct the study was granted by the Ethics and Research Committee of the New Territories West Cluster of the Hong Kong Hospital Authority. All the focus group members in the first part of the study and all the participants of the second part of the study had signed a written informed consent for their participation in this study.

\section{Focus groups}

Eight focus groups were held with 46 members to collect their views on information needs and participation needs of Chinese psychiatric inpatients. Members were inpatients of the acute wards $(n=8)$, medium-stay wards $(n=15)$, and rehabilitation wards $(n=10)$; patients' relatives $(n=6)$; and healthcare professionals $(n=7)$ of Castle Peak Hospital. Table 1 shows the socio-demographic characteristics of the focus group members.

In the focus group, group members discussed on the importance of information provision (e.g., patient charter, patient's rights and responsibilities, ward facilities, mental health educational materials related to mental illness and effects and side effects of medications, discharge information, hotlines services, and self-help groups) and patient's participation (e.g., in treatment plan, in the development of ground rules of wards, and in the improvement of the ward environment and services) for Chinese psychiatric inpatients. In the focus group discussion, the investigators acted as moderators and research assistants as co-moderators to guide the discussion according to a list of guiding questions and a list of non-directive probes as shown in the focus group discussion guide (Additional file 1).

In the focus group discussion, linguistic features were not the main focus. The discussion in fact served as a "verbal questionnaire" for exploring the group members' responses prompted by the guiding questions in the focus group discussion guide. Group members' responses were audio-taped and then transcribed by the investigator (MMYT, VCKL, HSML) of each group for analysis. In order to ensure the accuracy of the transcription, another investigator (NKYL, PHL, YLL) was responsible for checking the transcripts by reviewing the audio-tapes of each group.

The first step of analysis was to identify different items reflected by the transcripts concerning information and participation needs. An item could be a phrase, a sentence or a paragraph embodying ideas about information and participation needs. Starting from an inductive approach, the investigators (MMYT, VCKL, HSML, NKYL, PHL, YLL) read through the transcripts to identify the items that were relevant to information and participation needs and consensus was made in a face-to face group discussion among the investigators (MMYT, VCKL, HSML, NKYL, PHL, YLL). There had been no explicit criteria for rating an item as relevant and the investigators determined that an item was relevant in the face-to-face group discussion basing on their clinical experience. All the investigators had more than 5 years of clinical experience in psychiatric inpatient setting.

The second step followed a deductive approach by grouping the identified items to form categories with reference to the available literature. An initial categorisation system was then formulated by an investigator (VCKL). Transcripts of each of the focus groups were reviewed again by the investigator (MMYT, VCKL, HSML) of each group and another investigator (NKYL, PHL, YLL) to ensure the relevance and representativeness of the categorisation system for the 
Table 1 Demographic characteristics of focus group participants

\begin{tabular}{|c|c|c|}
\hline & Number & Percentage \\
\hline Professional group total & 7 & \\
\hline \multicolumn{3}{|l|}{ Gender } \\
\hline Male & 3 & 42.86 \\
\hline Female & 4 & 57.14 \\
\hline \multicolumn{3}{|l|}{ Professional group } \\
\hline Medical & 1 & 14.29 \\
\hline Nursing & 3 & 42.86 \\
\hline Occupational therapy & 1 & 14.29 \\
\hline Medical social work & 2 & 28.57 \\
\hline \multicolumn{3}{|c|}{ Years of experience in psychiatric services } \\
\hline $0-10$ years & 1 & 14.29 \\
\hline $11-20$ years & 3 & 42.86 \\
\hline$>20$ years & 3 & 42.86 \\
\hline Patient group total & 33 & \\
\hline \multicolumn{3}{|l|}{ Gender } \\
\hline Male & 21 & 64.10 \\
\hline Female & 12 & 35.90 \\
\hline \multicolumn{3}{|l|}{ Age } \\
\hline $18-30$ & 3 & 7.69 \\
\hline $31-40$ & 9 & 23.08 \\
\hline $41-50$ & 6 & 20.51 \\
\hline $51-60$ & 13 & 35.90 \\
\hline$>60$ & 2 & 12.82 \\
\hline \multicolumn{3}{|l|}{ Primary diagnosis } \\
\hline Schizophrenia & 28 & 87.18 \\
\hline $\begin{array}{l}\text { Depression/Bipolar affective } \\
\text { disorder/Schizoaffective disorder }\end{array}$ & 5 & 12.82 \\
\hline \multicolumn{3}{|l|}{ Education Level } \\
\hline No formal education & 1 & 5.13 \\
\hline Primary & 3 & 12.82 \\
\hline Secondary & 26 & 74.36 \\
\hline Tertiary & 3 & 7.69 \\
\hline \multicolumn{3}{|l|}{ Current wards/units } \\
\hline Acute wards & 8 & 23.08 \\
\hline Medium-stay wards & 15 & 41.03 \\
\hline Rehabilitation wards & 10 & 35.90 \\
\hline Relative group total & 6 & \\
\hline \multicolumn{3}{|l|}{ Gender } \\
\hline Male & 2 & 33.33 \\
\hline Female & 4 & 66.67 \\
\hline \multicolumn{3}{|l|}{ Age } \\
\hline $18-40$ & 0 & 0.00 \\
\hline $41-60$ & 4 & 66.66 \\
\hline$>60$ & 2 & 33.33 \\
\hline
\end{tabular}

Table 1 Demographic characteristics of focus group participants (Continued)

\begin{tabular}{lll}
\hline Education Level & & \\
No formal education & 0 & 0.00 \\
Primary & 2 & 33.33 \\
Secondary & 2 & 33.33 \\
Tertiary & 2 & 33.33 \\
Relationship with the patient & & \\
Patient's parent & 4 & 66.67 \\
Patient's child & 1 & 16.67 \\
Patient's sibling & 1 & 16.67 \\
\hline
\end{tabular}

content of the transcripts. A pool of items for information provision and patient's participation were identified. For each of the identified item, all the investigators agreed that the item was relevant/highly relevant and are representative/highly representative in describing either the information needs or participation needs of Chinese psychiatric inpatients.

\section{Development of survey questionnaire}

A total of 22 items of information needs and 16 items of participation needs of Chinese psychiatric inpatients were identified from the focus group discussion (Table 2).

Basing on the item pool obtained on information provision and patient's participation in the focus group discussion, a survey questionnaire was developed (Additional file 2).

\section{Validation of the survey questionnaire}

The survey questionnaire was developed basing on the focus group discussion among Chinese psychiatric inpatients, patients' relatives and healthcare professionals and its face validity with cultural adaptation was established. The content validity of the questionnaire was established by the agreements among investigators who were healthcare professionals on its relevance and representativeness in assessing the information and participation needs of Chinese psychiatric inpatients. Factor analysis was not performed for further testing the construct validity of the questionnaire and the test -retest reliability as well as the sensitivity to changes of the questionnaire were not tested in this study. The validity and reliability of the survey questionnaire are yet to be further explored in future studies with larger samples.

\section{Participants of questionnaire survey}

A group of patients were recruited from the acute, medium-stay, and rehabilitation wards of the Department of Adult Psychiatry and the Department of Forensic Psychiatry of Castle Peak Hospital $(\mathrm{CPH})$ over a 6-month period as participants to complete the survey questionnaire 
Table 2 Information needs and participation needs derived from focus group discussion

\begin{tabular}{ll}
\hline Information needs (22 items) & Participation needs (16 items) \\
\hline $\begin{array}{l}\text { Information on the classifications of } \\
\text { mental illnesses, signs and symptoms }\end{array}$ & $\begin{array}{l}\text { Collaborating with professionals } \\
\text { in setting treatment and care }\end{array}$ \\
$\begin{array}{l}\text { Information on the reasons for } \\
\text { in-patient admission and related }\end{array}$ & Learning and practising self- \\
ordinance & management
\end{tabular}

Information on different residential placements

Information on daytime training placements after discharge from in-patient setting

Information on the criteria and arrangements for discharge

Information on the ways to raise self-image and overcome stigma adhered to people with mental illnesses

Information on hospital fees and application of financial assistance

Information on the reasons behind the hospital rules and regulations

Information on the daily routine and schedules of ward

Information on how to maintain good physical health

Information on the arrangements at different stages of rehabilitation including pre-discharge arrangements

Information on the news from the outside world

Information on patient's right (including ways of expressing opinion and making enquiries)

Information on how relatives can accept, participate and help

Information on job acquisition

Information on stress management and relapse prevention

Enquiring about personal needs and arrangements

Providing opinion on how to improve the services and facilities of ward

Keeping in touch with the outside world

Expressing needs and following up whether needs are meet

Understanding the treatment and rehabilitation plans and providing enough information for healthcare professionals to take reference

Learning from other patients

Reporting discomfort actively

Expressing personal feelings

Preparing for the interview with healthcare professionals

Learning how to cope with stress and prevent relapse

Participating in vocational training

Participating in social activities

Participating in training of domestic and self-care skill

Learning how to take care of others

Information on alcohol and substance misuse

Information on the schedule of meetings with healthcare professionals

Information on community services and resources

Information on the different kinds of care and rehabilitation services

Information on medication prescriptions

Information on the importance of psychiatric drug taking and its side effects
(Additional file 2). The inclusion criteria were ethnic Chinese aged 18 to 64 years and were able and willing to provide written informed consent to participate in the study. Those with moderate grade mental deficiency and were not able to comprehend Chinese were excluded. All the participants had signed a written informed consent for the participation in this study. Socio-demographic and clinical characteristics of the participants were obtained.

In the survey questionnaire, the participants were asked to choose ten items of information provision and ten items of patient's participation that were perceived by them as the most important. They were then asked to arrange the items in rank order with one representing the most important and ten representing the least important among the ten important items for information provision and patient's participation.

\section{Statistical analysis}

Survey data were analysed with the Statistical Package for Social Sciences, Windows version 17.0. Descriptive statistics were applied for data analysis of the questionnaire survey and Chi-square tests with cross tabulation were adopted to explore whether there were any differences across the results of the questionnaire survey in terms of gender, age group, educational level, and diagnosis of the participants. Significant levels were set at $p<0.05$ throughout the study.

\section{Results}

\section{Survey on needs of psychiatric inpatients}

A convenience sample of 183 inpatients recruited from the acute wards, medium-stay wards, and rehabilitation wards of $\mathrm{CPH}$ completed the survey questionnaire on the importance of different items concerning information and participation needs. More than half $(60.1 \%)$ of the inpatients were males. The largest group by age $(29.0 \%)$ fell into the age range of $31-40$ years and most $(68.3 \%)$ of the total sample had a diagnosis of schizophrenia (Table 3).

\section{Information needs}

For information needs, the top five needs rated by patients as the most important in descending order were: "Information on the classifications of mental illnesses, signs and symptoms and factors contributing to relapse" (frequency of being rated as important: 125), "Information on the criteria and arrangements for discharge" (frequency of being rated as important: 112), "Information on the importance of psychiatric drug taking and its side effects" (frequency of being rated as important: 109), "Information on hospital fees and application of financial assistance" (frequency of being rated as important: 107), and "Information on daytime training placements after 
Table 3 Demographic characteristics of participants in the questionnaire survey

\begin{tabular}{|c|c|c|}
\hline & Number & Percentage \\
\hline Total & 183 & 100 \\
\hline \multicolumn{3}{|l|}{ Gender } \\
\hline Male & 110 & 60.11 \\
\hline Female & 73 & 39.89 \\
\hline \multicolumn{3}{|l|}{ Age } \\
\hline $18-30$ & 39 & 21.31 \\
\hline $31-40$ & 53 & 28.96 \\
\hline $41-50$ & 45 & 24.59 \\
\hline $51-60$ & 41 & 22.40 \\
\hline$>60$ & 5 & 2.73 \\
\hline \multicolumn{3}{|l|}{ Primary Diagnosis } \\
\hline Schizophrenia & 125 & 68.31 \\
\hline $\begin{array}{l}\text { Depression/Bipolar affective } \\
\text { disorder/Schizoaffective disorder }\end{array}$ & 58 & 31.69 \\
\hline \multicolumn{3}{|l|}{ Education Level } \\
\hline No formal education & 5 & 2.73 \\
\hline Primary & 28 & 15.30 \\
\hline Secondary & 141 & 77.05 \\
\hline Tertiary & 9 & 4.92 \\
\hline \multicolumn{3}{|l|}{ Current wards/units } \\
\hline Acute wards & 35 & 19.13 \\
\hline Medium-stay wards & 93 & 50.82 \\
\hline Rehabilitation wards & 55 & 30.05 \\
\hline
\end{tabular}

discharge from inpatient setting" (frequency of being rated as important: 95) (Table 4).

There were no significant differences between different genders (male vs female) on the ratings of the items on information needs. Concerning the ratings by different age groups (aged 40 years or below vs aged $>40$ years), significantly more participants in the younger age group rated "Information on medication prescriptions" as important $\left(\chi^{2}(1, N=183)=22.44, p=.004\right)$. For ratings by different educational groups (primary educational level or less vs secondary educational level or above), significantly more participants with a lower educational level rated "Information on the daily routine and schedules of ward" as important $\left(\chi^{2}(1, N=183)=18.88, p=.026\right)$. For diagnostic groups (schizophrenia vs non-schizophrenia), significantly more participants with a diagnosis of schizophrenia rated "Information on the importance of psychiatric drug taking and its side effects" as important $\left(\chi^{2}(1, N=183)=17.32, p=.044\right)$.

\section{Participation needs}

For participation needs, the top five needs rated by patients as the most important in descending order were:
Table 4 Information needs

\begin{tabular}{|c|c|c|}
\hline Information needs (22 items) & $\begin{array}{l}\text { Frequency } \\
(n=183)\end{array}$ & $\begin{array}{l}\text { Frequency } \\
\text { rank }\end{array}$ \\
\hline $\begin{array}{l}\text { Information on the classifications of mental } \\
\text { illnesses, signs and symptoms and factors } \\
\text { contributing to relapse }\end{array}$ & 125 & 1 \\
\hline $\begin{array}{l}\text { Information on the reasons for in-patient } \\
\text { admission and related ordinance }\end{array}$ & 93 & 6 \\
\hline Information on different residential placements & 90 & 10 \\
\hline $\begin{array}{l}\text { Information on daytime training placements } \\
\text { after discharge from in-patient setting }\end{array}$ & 95 & 5 \\
\hline $\begin{array}{l}\text { Information on the criteria and arrangements } \\
\text { for discharge }\end{array}$ & 112 & 2 \\
\hline $\begin{array}{l}\text { Information on the ways to raise self-image } \\
\text { and overcome stigma adhered to people } \\
\text { with mental illnesses }\end{array}$ & 93 & 7 \\
\hline $\begin{array}{l}\text { Information on hospital fees and application } \\
\text { of financial assistance }\end{array}$ & 107 & 4 \\
\hline $\begin{array}{l}\text { Information on the reasons behind the hospital } \\
\text { rules and regulations }\end{array}$ & 65 & \\
\hline $\begin{array}{l}\text { Information on the daily routine and schedules } \\
\text { of ward }\end{array}$ & 76 & \\
\hline $\begin{array}{l}\text { Information on how to maintain good physical } \\
\text { health }\end{array}$ & 91 & 9 \\
\hline $\begin{array}{l}\text { Information on the arrangements at different } \\
\text { stages of rehabilitation including pre-discharge } \\
\text { arrangements }\end{array}$ & 82 & \\
\hline $\begin{array}{l}\text { Information on the news from the outside } \\
\text { world }\end{array}$ & 72 & \\
\hline $\begin{array}{l}\text { Information on patient's right (including ways } \\
\text { of expressing opinion and making enquiries) }\end{array}$ & 93 & 8 \\
\hline $\begin{array}{l}\text { Information on how relatives can accept, } \\
\text { participate and help }\end{array}$ & 76 & \\
\hline Information on job acquisition & 87 & \\
\hline $\begin{array}{l}\text { Information on stress management and relapse } \\
\text { prevention }\end{array}$ & 81 & \\
\hline Information on alcohol and substance misuse & 42 & \\
\hline $\begin{array}{l}\text { Information on the schedule of meetings with } \\
\text { healthcare professionals }\end{array}$ & 60 & \\
\hline $\begin{array}{l}\text { Information on community services and } \\
\text { resources }\end{array}$ & 57 & \\
\hline $\begin{array}{l}\text { Information on the different kinds of care and } \\
\text { rehabilitation services }\end{array}$ & 46 & \\
\hline Information on medication prescriptions & 59 & \\
\hline $\begin{array}{l}\text { Information on the importance of psychiatric } \\
\text { drug taking and its side effects }\end{array}$ & 109 & 3 \\
\hline
\end{tabular}

"Enquiring about personal needs and arrangements" (frequency of being rated as important: 142), "Keeping in touch with the outside world" (frequency of being rated as important: 135), "Learning and practising selfmanagement" (frequency of being rated as important: 132), "Collaborating with professionals in setting treatment and care plans" (frequency of being rated as important: 129), 
and "Reporting discomfort actively" (frequency of being rated as important: 121) (Table 5).

There were no significant differences between different genders (male vs female) and different educational groups (primary educational level or less vs secondary educational level or above) on the ratings of the items on participation needs. Concerning the ratings by different age groups (aged 40 years or below vs aged $>40$ years), significantly more participants in the younger age group rated "Keeping in touch with the outside world" as important $\left(\chi^{2}(1, N=183)=23.85, p=.005\right)$. For diagnostic groups (schizophrenia vs non-schizophrenia), significantly more participants with a diagnosis of schizophrenia rated "Understanding the treatment and rehabilitation plans and providing enough information for healthcare professionals to take reference" as important $\left(\chi^{2}(1, N=183)=24.05\right.$, $p=.004)$.

\section{Discussion}

\section{Recovery-oriented mental health service}

In the implementation of recovery-oriented mental health service, "patient's empowerment with the provision of information" and "patient's participation' should be the

Table 5 Participation needs

\begin{tabular}{lll}
\hline Participation needs (16 items) & $\begin{array}{l}\text { Frequency } \\
(n=183)\end{array}$ & $\begin{array}{l}\text { Frequency } \\
\text { rank }\end{array}$ \\
\hline $\begin{array}{l}\text { Collaborating with professionals in setting } \\
\text { treatment and care plans }\end{array}$ & 129 & 4 \\
Learning and practising self-management & 132 & 3 \\
$\begin{array}{l}\text { Enquiring about personal needs and } \\
\text { arrangements }\end{array}$ & 142 & 1 \\
$\begin{array}{l}\text { Providing opinion on how to improve the } \\
\text { services and facilities of ward }\end{array}$ & 107 \\
$\begin{array}{l}\text { Keeping in touch with the outside world } \\
\text { Expressing needs and following up whether }\end{array}$ & 135 \\
$\begin{array}{l}\text { needs are meet } \\
\text { Understanding the treatment and rehabilitation } \\
\text { plans and providing enough information for } \\
\text { healthcare professionals to take reference }\end{array}$ & 95 & 2 \\
Learning from other patients & 91 & 6 \\
$\begin{array}{l}\text { Reporting discomfort actively } \\
\text { Expressing personal feelings }\end{array}$ & 121 \\
$\begin{array}{l}\text { Preparing for the interview with healthcare } \\
\text { professionals }\end{array}$ & 90 \\
$\begin{array}{l}\text { Learning how to cope with stress and prevent } \\
\text { relapse }\end{array}$ & 106 & 5 \\
$\begin{array}{l}\text { Participating in vocational training } \\
\text { Participating in social activities }\end{array}$ & 116 \\
$\begin{array}{l}\text { Participating in training of domestic and } \\
\text { self-care skill } \\
\text { Learning how to take care of others }\end{array}$ & 111 & 7 \\
\hline
\end{tabular}

strategies that are promoted by all those involved in care delivery. Exploration of the information needs and participation needs of Chinese psychiatric inpatients should be an important step for the implementation of recovery-oriented mental health service. This qualitative and quantitative study investigated the information needs and participation needs of Chinese psychiatric inpatients.

\section{Information needs}

Results of this study revealed that "Information on the classifications of mental illnesses, signs and symptoms and factors contributing to relapse", "Information on the criteria and arrangements for discharge", "Information on the importance of psychiatric drug taking and its side effects", "Information on hospital fees and application of financial assistance", and "Information on daytime training placements after discharge from in-patient setting" were rated as the most important information that Chinese psychiatric in-patients perceived that they needed to have during their hospital stay.

Surveys reveal that meeting the information needs and participation needs of patients increases their satisfaction for the services $[9,10,19,20]$. Moreover, providing psychiatric patients with information about treatment and involving them in care decisions during inpatient care may help to facilitate the transition from inpatient to outpatient settings [30]. On the other hand, inadequacy of information and the consequent exclusion from discussions and decisions about treatment are considered as enduring complaints by service users about mental health services [18].

A recent patient satisfaction survey in Castle Peak Hospital pointed out the needs for further improvement in information provision and participation [31]. The survey also highlighted the concern about health information available to patients on both the quantity of information (e.g., amount of information, number of sources, types and strategies for distribution) and the quality of information (e.g., validity, relevance, accessibility, understandability, timing of acquisition) [31].

The results of the current study were in line with the findings of studies in other countries on non-Chinese populations [32-34]. In a study on the educational needs of chronic psychiatric patients, it was found that patients reported strong interest in learning more about psychiatric illness [32]. In another study on the quality of psychiatric inpatient care, conducted in the United Kingdom, it was found that providing patients with information on health conditions, psychiatric medications, and care after discharge was important in the delivery of high quality mental health care [33]. A survey conducted in Germany on the indicators of quality of inpatient psychiatric treatment from the patients' view revealed that information about the effects and side effects of medications as well as the 
effectiveness of medications were rated as a significantly important indicator [34]. The results of the current study also showed that patients with different age groups, educational levels, and diagnoses had different information needs so the provision of information should be based on the different socio-demographic and clinical characteristics of the patients. The current study showed that younger patients were more concerned about information on the medications prescribed to them. Younger patients in this study may be in an earlier stage of mental illness so that they may be more concerned about the information on the medications which they need to be taken for the subsequent months or years. In the current study, patients with lower educational level rated the information on the daily routine and schedule of ward as important to them. This result indicates that patients with lower educational level may be more concerned about basic ward information such as the time for waking up and going to bed as well as time for meals and bathing whereas those with higher educational level may require more information on their mental illness and medications. Also, patients with schizophrenia were more concerned about the side effects of the medications prescribed to them. This result can be explained by the obvious potential side effects of antipsychotics such as extra-pyramidal side effects as compared with that caused by medications for treatment for other diagnoses such as antidepressant for depression.

The results of the present study serve as a reference for the design of information materials in order to meet the information needs of Chinese psychiatric inpatients. As for the delivery of information in psychiatric inpatient setting, it is certainly a task for multi-disciplinary input. For examples, case medical officers provide information on medications; nurses provide information on mental health; occupational therapists provide information on vocational placements; and medical social workers provide information on financial support. Psychiatric nurses have a significant role to take in the empowering process due to their long duration of contact with the patients in day-to-day nursing care at wards, while the other professionals also play very important roles to empower patients in different degrees during patient contacts.

\section{Participation needs}

The participation and involvement of service users in organisational service planning and services' clinical governance processes has been highlighted in the literature on quality of psychiatric inpatient care [8]. In addition to collaboration in their care, service users' involvement in developing, running and reviewing services has been shown to have positive outcomes for both the services and the service users who take on these roles $[9,10]$. Chinese psychiatric patients are relatively passive and seldom speak up and express their needs. In contrast, traditional Chinese mental health professionals tend to adopt the "paternalistic" or "maternalistic" model of care and design care plans for patients based on the professionals' own perspective [24]. Patients' participation in their care in psychiatric inpatient settings is therefore a challenge for both patients and mental health professionals in the Chinese populations.

Results of this study revealed that "Enquiring about personal needs and arrangements", "Keeping in touch with the outside world", "Learning and practising self-management", "Collaborating with professionals in setting treatment and care plans", and "Reporting discomfort actively" were rated as the most important participation needs for Chinese psychiatric inpatients. These results showed that despite Chinese psychiatric inpatients being relatively passive, they still rated collaboration with healthcare professionals in deriving their treatment and care plan as important. The results of the current study were in line with other studies on non-Chinese populations that psychiatric inpatients do consider that their participation in their treatment plan as important [32, 34-36]. In a study conducted in America on shared decision-making preferences of patients with severe mental illness, most of the patients preferred shared decision making, particularly in relation to their mental health care [35]. Another study conducted in America on the need of chronic psychiatric inpatients revealed that patients had strong interest in learning strategies and skills for coping with common personal problems [32]. A study conducted in Germany showed that patient's participation in medication decisions was rated as a significant indicator for high quality psychiatric inpatient care [34]. In contrast to other studies on nonChinese populations in which psychiatric patients wanted a more active role in psychiatric service planning and policy making, the Chinese patients in our present study were concerned much more with their personal needs and selfmanagement, as well as their participation in their own treatment plan [37].

Basing on the results of the current study, the participation needs of Chinese psychiatric inpatient might be met by making posters, pamphlets, videos to encourage patients to speak up and participate, by encouraging patients to join multi-disciplinary clinical case round, and by involving patients in the early stage of care plan compilation. On the other hand, training should be provided to patients on their self-management skills and domestic skills. The results of the current study showed that patients from different age groups and patients with different psychiatric diagnoses had different participation needs and a significant number of patients, especially those with a younger age, rated keeping in touch with the outside world as important. Despite there has been no study on Chinese populations showing similar finding, the finding in this study that younger patients wanted to keep 
in touch with the outside world can be explained by the rapid developments of information technology such as internet and facebook that patients, especially those with younger age, would like to continue to get access to different information that they could have access to before they are hospitalised. Therefore, inpatient programmes should be tailored made according to the different socio-demographic and clinical characteristics of inpatients such as their age groups and diagnostic groups and should also include community orientation and news reading or internet surging in order to keep the inpatients in touch with the outside world. The current study also revealed that patients with schizophrenia were more concerned about participation in their rehabilitation plan. Despite there has been no similar result found in the Chinese populations in previous studies, this result highlighted the importance of involving schizophrenia patients in the design and revision of their rehabilitation plan.

\section{Limitations and suggestions for future research}

This study had several limitations. The sample size was small and only adult Chinese inpatients in a mental hospital were recruited with the exclusion of child and adolescent patients and elderly patients. Generalisability of the study is therefore limited. Patients rated the importance of their information needs and participation needs basing on a questionnaire with a fixed list of items identified by focus group discussion. It might have been worthwhile to allow patients to write in the questionnaire in an open-ended format if they think that there are any other areas of information needs and participation needs that are important but are not addressed in the list in the questionnaire. A future study with a larger sample size is warranted in order to analyse whether Chinese inpatients from acute wards, medium-stay wards, and rehabilitation wards have different information and participation needs. This was a cross-sectional study with the inpatients rated their information and participation needs at one particular time point of their illness stage. A longitudinal study could be conducted to follow up the patients throughout their pathway of inpatient care to explore how their information and participation needs change as they go through different stages of their mental illness (e.g., at the acute, subacute, and chronic stages of illness) and its treatment. Moreover, the psychometric properties of the survey questionnaire were not thoroughly tested in the present study. It would be worthwhile to recruit a larger sample in order to perform factor analysis or Rasch analysis as well as test-retest reliability analysis for the exploration of the validity and reliability of the survey questionnaire in future. A longitudinal study is also warranted to assess the sensitivity to changes of the survey questionnaire.

\section{Conclusions}

The concept and construct of recovery, despite being rather abstract, has been evident in patient's self-help since the 1930s and gained its prominence in the mental health literature since the 1980s [38]. The adoption of the concept of recovery in mental health service transformation and delivery has been widely discussed in the literature in the recent years [39-41]. The incorporation of the recovery principles into the government reports and policy statements on mental health service in many countries as well as the rapid emergence of studies on mental health recovery point to the urgent need to explore culturally-relevant ways of implementing mental health recovery-oriented practice within Chinese populations $[1-7,11,42-46]$. Apart from being in line with the global trend of mental health service reform, the implementation of recovery-oriented practices also matches well with the increasing emphasis of human rights and patient's involvements in the Chinese society [24]. Meeting the information needs and participation needs of psychiatric inpatients are salient for the delivery of quality psychiatric inpatient care and is proven to improve patients' outcomes. This is the first study to explore the information needs and participation needs of Chinese psychiatric inpatients in this locality. This study reveals that Chinese psychiatric inpatients are concerned about information on their mental illness and its treatments as well as the criteria for discharge. On the other hand, patients are concerned about their personal needs, their selfmanagement, as well as their keeping in touch with the outside world during their hospitalisation. Moreover, patients with different socio-demographic and clinical characteristics have different information and participation needs. The results of the present study serve as a reference for mental health professionals and policy makers to design strategies, guidelines, service models, and programmes in order to fulfil the information needs and participation needs of Chinese psychiatric inpatients in Hong Kong.

\section{Additional files}

Additional file 1: Focus Group Discussion Guide. This guide included a list of guiding questions and a list of non-directive probes to guide the focus group discussion. (DOCX $18 \mathrm{~kb}$ )

Additional file 2: Survey Questionnaire on Information and Participation needs. This survey questionnaire was developed basing on the item pool obtained on information provision and patient's participation in the focus group discussion and was the assessment tool adopted in the second part of the study. (DOCX $19 \mathrm{~kb}$ )

\section{Acknowledgements}

We would like to thank all the patients, their relatives, and staff of Castle Peak Hospital who have kindly participated in this study. We would also like to thank Dr. Eric Cheung, Dr. CW Cheng, Dr. M Lam, Dr. KM Cheng, Dr. KL Chung, and Dr. Henry Cheung for their unfailing support and advice for the conduction, implementation, and promulgation of this study. 


\section{Funding}

No specific funding was provided for this study.

\section{Availability of data and materials}

The dataset and materials supporting the conclusions of this article are included within the article and its additional files. Data can also be provided upon request.

\section{Authors' contributions}

BWMS made the study design, conceptualized the report, did the data interpretation, and wrote the report. MMYT, VCKL, HSML, NKYL, PHL, YLL made the study design, conceptualized the report, did the data collection and the data analysis. ACYL made the study design and critical appraisals of the report. ST made critical appraisals of the report. All authors read and approved the final manuscript

\section{Competing interests}

The authors declare that they have no competing interests.

\section{Consent for publication}

Not applicable.

\section{Ethics approval and consent to participate}

Written informed consent was signed by every participant participated in the present study. Participants were informed that they could withdraw from the study at any time. Approval to conduct the study was granted by the Ethics and Research Committee of the New Territories West Cluster of the Hong Kong Hospital Authority.

\section{Author details}

'Department of Psychiatry, Castle Peak Hospital, Hong Kong, SAR, People's Republic of China. ${ }^{2}$ The Mental Health Association of Hong Kong, Hong Kong, SAR, People's Republic of China. ${ }^{3}$ Department of Social Work and Social Administration, Associate Dean at Faculty of Social Sciences, The University of Hong Kong, Hong Kong, SAR, People's Republic of China. ${ }^{4}$ Department of Forensic Psychiatry, Castle Peak Hospital, 15 Tsing Chung Koon Road, Tuen Mun, New Territories, Hong Kong.

\section{Received: 23 December 2015 Accepted: 4 July 2016}

\section{Published online: 12 July 2016}

\section{References}

1. U.S. Department of Health and Human Services, Substance Abuse and Mental Health Services Administration. National Consensus Statement on Mental Health Recovery. 2004. Website: http://download.ncadi.samhsa.gov/ ken/pdf/SMA05-4129/trifold.pdf. Accessed 28 Aug 2011.

2. Commonwealth of Australia. National Standards for Mental Health Services. 2010. Website: http://www.health.gov.au/internet/mhsc/publishing.nsf/ Content/3545C977B46C5809CA25770D00093C93/\$File/NStds4mhs.pdf. Accessed 28 Aug 2011

3. Townsend W, Boyd S, Griffin G, Hicks PL. Emerging Best Practices in Mental Health Recovery. Ohio: Ohio Department of Mental Health; 2000.

4. Mental Health Commission of Canada. Toward Recovery \& Well-Being: A Framework for a Mental Health Strategy for Canada. 2009. Website: http://www.mentalhealthcommission.ca/SiteCollectionDocuments/Key_ Documents/en/2009/Mental_Health_ENG.pdf. Accessed 28 Aug 2011.

5. National Institute for Mental Health in England. Emerging Best Practice in Mental Health Recovery. UK: NIMHE; 2004.

6. NHS Education for Scotland/Scottish Recovery Network. Realising Recovery: a National Framework for Learning and Training in Recovery Focused Practice. Edinburgh: NES/SRN; 2007. Website: http://www.nes.scot.nhs.uk/media/ 5854/realisingrecoveryframeworkdocumentfinal.pdf. Accessed 28 Aug 2011.

7. Mental Health Commission. Blueprint for Mental Health Services in New Zealand. Wellington: Mental Health Commission; 1998.

8. Taylor TL, Killaspy H, Wright $\mathrm{C}$, et al. A systematic review of the international published literature relating to quality of institutional care for people with longer term mental health problems. BMC Psychiatry. 2009;9:55.

9. Linhorst DM, Eckert A. Involving people with severe mental illness in evaluation and performance improvement. Eval Health Prof. 2002;25:284-301.

10. Ahuja AS, Williams R. Involving patients and their carers in educating and training practitioners. Curr Opin Psychiatry. 2005;18:374-80.
11. Anthony WA. Recovery from mental illness: the guiding vision of the menta health service system in the 1990s. Psychosoc Rehabil J. 1993;16:11-23.

12. Farkas M, Gagne C, Anthony W, et al. Implementing recovery-orientated evidence-based programmes: identifying the critical dimensions. Community Ment Health J. 2005;42(2):141-58.

13. Leamy M, Bird V, Clair LB, Williams J, Slade M. Conceptual framework for personal recovery in mental health: systematic review and narrative synthesis. Br J Psychiatry. 2011;199:445-52.

14. Slade M. Personal recovery and mental illness. A guide for mental health professionals. Cambridge: Cambridge University Press; 2009.

15. Davidson L, Tondora J, Lawless MS, O'Connell M, Rowe M. A practical guide to recovery-oriented practice tools for transforming mental health care. USA: Oxford University Press; 2009.

16. Kane JR. Information is the key to patient empowerment. Annals Health L. 2002;11:25.

17. Dey A. Consumer health informatics: an overview of patient perspectives on health information needs. HIMJ. 2004:33:121-6.

18. Pollock K, Grime J, Baker E, Mantala K. Meeting the information needs of psychiatric inpatients: staff and patient perspective. JMH. 2004;13:389-401.

19. Hansson L, Björkman T, Berglund I. What is important in psychiatric inpatient care? Quality of care from the patient's perspective. Qual Assur Health Care. 1993;5:41-7

20. Mccann TV, Baird J, Clark E, Lu S. Mental health professionals' attitude towards consumer participation in inpatient units. J Psychiatr Ment Health Nurs. 2008;15:10-6.

21. Siu BWM, Sung RWK. Promotion and Implementation of Recovery-oriented Practice in a Mental Hospital in Hong Kong. Hong Kong J Ment Health. 2013;39:13-9.

22. Chou LF. The ecology of mental health care in Taiwan. Adm Policy Ment Health. 2006:33(4):492-8.

23. Yu X, Liu J, Ma H. Community mental health: a panacea to confront the challenge in China? [in Chinese]. Chin Ment Health J. 2010;24:885-6.

24. Liu J, Ma H, He Y-L, et al. Mental health system in China: history, recent service reform and future challenges. World Psychiatry. 2011;10:210-6.

25. Ma H. Integration of hospital and community services - the "686 Project" - is a crucial component in the reform of China's mental health services. Shanghai Arch Psychiatry. 2012;24:172-4.

26. Tse S, Ran MS, Huang Y, Zhu S. Mental Health Care Reforms in Asia: The urgency of now: building a recovery-oriented, community mental health service in China. Psychiatr Serv. 2013;64(7):613-6.

27. Tse S, Siu BWM, Kan A. Can Recovery-Oriented Mental Health Services be Created in Hong Kong? Struggles and Strategies. Adm Policy Ment Health. 2013:40:155-8

28. Siu BWM, Yeung YM, Poon MY, Sun LNN. Attitude towards Recovery in Chinese Mental Health Professionals. Hong Kong J Ment Health. 2011;37 35-42.

29. Siu BWM, Ng BFL, Li VCK, Yeung YM, Lee MKL, Leung AYH. Mental Health Recovery for Psychiatric In-patient Services - Perceived Importance of the Elements of Recovery. East Asian Arch Psychiatry. 2012;22:39-48.

30. Bowersox NW, Bohnert AS, Ganoczy D, Pfeiffer PN. Inpatient psychiatric care experience and its relationship to posthospitalization treatment participation. Psychiatr Serv. 2013;64:554-62.

31. Fu JCK, Chow PPL, Lam JWS, Tung RCK. Patient Satisfaction Survey, Castle Peak Hospital. Hong Kong SAR: Castle Peak Hospital; 2011.

32. Mueser KT, Bellack AS, Wade JH, Sayers SL, Rosenthal CK. An assessment of the educational needs of chronic psychiatric patients and their relatives. Br J Psychiatry. 1992;160:674-80.

33. Gigantesco A, Morosini P, Bazzoni A. Quality of psychiatric care; validation of an instrument for measuring inpatient opinion. Int J Qual Health Care 2003;15:73-8.

34. Längle G, Baum W, Wollinger A, Renner G, Úren R, Schwärzler F, Eschweiler GW Indicators of quality of in-patient psychiatric treatment: the patients'view. Int J Qual Health Care. 2003;15:213-21.

35. Adams JR, Drake RE, Wolford GL. Shared decision-making preferences of people with severe mental illness. Psychiatr Ser. 2007;58:1219-21.

36. Henderson J, Curren D, Walter B, Toffoli L, O'Kane D. Relocating care: negotiating nursing skillmix in a mental health unit for older adults. Nurs Inq. 2011:18(1):55-65.

37. Glenister D. Patient participation in psychiatric services: a literature review and proposal for a research strategy. J Adv Nurs. 1994;19:802-11.

38. Ralph RO. Recovery. Psychiatr Rehab Skills. 2000;4:480-517. 
39. Ridgway P, Press A. Assessing The Recovery - Commitment of Your Mental Health Service: A User's Guide For The Developing Recovery Enhancing Environments Measure (DREEM). 2004. Website: http://64.233.169.104/ search?q=cache:mB9Ag810-SMJ:www.outcomemeasures.csip.org.uk/silo/ files/dreem-.pdf+DREEM, + ridgway, + press\&hl=en\&ct=clnk\&cd=3\&gl=us. Accessed 28 Aug 2011.

40. Armstrong NP, Steffen JJ. The recovery promotion fidelity scale: assessing the organizational promotion of recovery. Community Ment Health J. 2009:45:163-70.

41. Houselink Community Homes. Recovery Survey DREEM. 2010. Website: http://new.houselink.on.ca/wp-content/uploads/2011/03/2010-DREEMSurvey.pdf. Accessed 28 Aug 2011.

42. Deegan PE. Recovery as a journey of the heart. Psychiatr Rehabil J. 1996;19: 91-7.

43. Deegan PE. Recovery: The lived experience of rehabilitation. Psychosoc Rehabil J. 1988;11:11-9.

44. Spaniol L, Gagne C, Koehler M. Recovery from serious mental illness: What it is and how to assist people in their recovery. Continuum. 1997;4:3-15.

45. Killaspy $\mathrm{H}$, White $\mathrm{S}$, Taylor TL, King M. Psychometric properties of the Mental Health Recovery Star. Br J Psychiatry. 2012;201:65-70. doi:10.1192/bjp.bp.111. 107946

46. Stocks ML. In the eye of the beholder. Psychiatr Rehabil. 1995;19:89-91.

\section{Submit your next manuscript to BioMed Central} and we will help you at every step:

- We accept pre-submission inquiries

- Our selector tool helps you to find the most relevant journal

- We provide round the clock customer support

- Convenient online submission

- Thorough peer review

- Inclusion in PubMed and all major indexing services

- Maximum visibility for your research

Submit your manuscript at www.biomedcentral.com/submit 